\title{
Study on New Thermal Drying Methods for Sewage Sludge Using Microwave and its Mechanism
}

\author{
Li Jinping ${ }^{1}, a^{*}$, Gan Jinhua ${ }^{2}$, b, Hu Jieqiong ${ }^{1}$, c, Wang Ni ${ }^{1}$, d \\ ${ }^{1}$ School of Environmental Engineering, Wuhan Textile University, Wuhan, 430073, China \\ ${ }^{2}$ Yangtze River Fisheries Research Institute, Wuhan, 430050, China

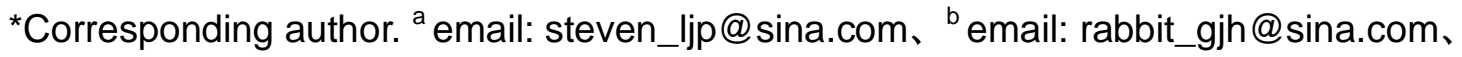 \\ cemail:381550699@qq.com、demail: wangni@163.com
}

\begin{abstract}
Key words: sewage sludge; microwave drying; drying technology; drying rate
Abstract: The paper mainly targets at investigating which municipal sludge drying technology is efficient and economical. Using the drying method of microwave to study on the factors influenced over the drying efficiency of municipal sludge. And do the experiment analysis and comparison about the energy consumption of microwave methods to seek for the more efficient, energy-saving sludge drying technology. The results show that Sludge microwave drying process consists of three stages: acceleration phase, constant speed phase and deceleration phase. During the microwave drying process, when the microwave power is the same, the bigger diameter of the municipal sludge is the faster drying rate exists. The higher the microwave power, the faster drying rate exists. In this research, the unit energy consumption of $480 \mathrm{~W}$ microwave power is lower than others, and the drying rate in this condition is relatively faster. The research conclusions will provide a scientific basis for municipal sludge drying and semi-drying in engineering practice, which has a certain guiding significance.
\end{abstract}

\section{Introduction}

Sludge is a kind of solid, semisolid or liquid residue produced in the process of sewage treatment ${ }^{[1-3]}$.In order to accurately reflect the fact that the most of sewage sludge owns re-utilized value, The Water Environment Federation(WEF) renamed "Sewage Sludge" as "Biosolids" "[4], which marks the transformation and maturity of the disposal concept of sewage sludge in a certain extent, thus the sludge could get into the ecosystem nutrient cycling.

Within a year dry sludge produced can be up to tens of millions of tons in the whole world, mainly in developed countries, such as America, Europe, Japan and so on ${ }^{[5-6]}$. According to the estimating of the amount of municipal wastewater and the production of sludge in the city life, the dry sludge production of 2.08 million tons in 2005 is much higher than the forecast. In accordance with the rapid development of the sewage treatment industry in China, what can be expected in the next ten year is that the production of sludge will be greatly increased, and the disposal of sludge will become a more prominent environmental problem in our country ${ }^{[7-8]}$.

Microwave is a kind of electromagnetic wave of $3 \times 10^{2} \sim 3 \times 10^{5} \mathrm{MHz}$.Rrsently the microwave is used as an energy source for heating, drying and simulating plasma, which expands another branch of microwave utilization. Microwave heating has several notable features: instantaneous, selective and penetrating. The research shows that microwave heating has a significant effect on the drying efficiency. And this study provides not only a new technical reference and theoretical support for the sludge of drying and sludge of semi-drying, but also a scientific basis for engineering practice of microwave drying of sludge by the specialty of microwave heating drying sludge ${ }^{[9-11]}$. 


\section{Materials and methods}

\section{Experimental materials and apparatus}

The sludge comes from the sludge dewatering room of sewage treatment plant in Wuhan, which was disposed by the belt filter dewatering, and the average water content of sludge was $82.30 \%$.The microwave source used in the experiment is from the G80F20CSL-B8 (R0) microwave oven, and its working frequency is $2450 \mathrm{MHz}$, which has 5 file can be adjusted. The scale is the HX1201Z electronic balance (minimum of $0.1 \mathrm{~g}$ ). The electric heating air-blowing drier of ST368-1 type is used in the experiment.

\section{Experimental method}

Specific experimental method: Make fresh sewage sludge into sludge ball/sludge cake with diameter and diameter, which was used in microwave drying. In the microwave conditions of 320W, $480 \mathrm{~W}, 640 \mathrm{~W}$ and $800 \mathrm{~W}$, multi-group sludge ball/sludge cake was dried and the drying rate curve of sludge was calculated and drew by measuring the weight of sludge at different times.

\section{Results and discussion}

\section{The effect of different microwave power on the drying rate of sludge}

First, make sludge into sludge cake with $3.0 \mathrm{~cm}$ in diameter and $1.0 \mathrm{~cm}, 2.0 \mathrm{~cm}$ and $3.0 \mathrm{~cm}$ in thickness, and each of these are two. Then puts this sludge into the six clean crucible which is burned to the constant weight. Third, under the microwave condition of $160 \mathrm{~W}$, the mass of the sludge cake was quickly weighed at every moment (according to the size of mud cake and the power to adjust). When the moisture content reached $2 \%$, the experiment was stopped. The experiment was repeated under microwave conditions of $320 \mathrm{~W}, 480 \mathrm{~W}, 640 \mathrm{~W}$ and $800 \mathrm{~W}$.Last, under different microwave conditions, calculate the moisture content and drying rate of different thickness of mud cake at different time points, and draw the sludge drying rate curve, as shown in figure 1, figure 2 , figure 3 , figure 4 , figure 5 .

By Figure1,under the microwave condition of $180 \mathrm{~W}$, the shape of the dry rate curve of the sludge cake with the diameter of $3.0 \mathrm{~cm}$ and the thickness of $1.0 \mathrm{~cm}, 2.0 \mathrm{~cm}, 3.0 \mathrm{~cm}$ is consistent, which is comprise of three phases, that is acceleration $(70.0 \%<\mathrm{P}<83.3 \%)$, constant speed $(50.0 \%<\mathrm{P}<70.0 \%)$ and deceleration $(\mathrm{P}<50.0 \%)$.From the picture, when the moisture content of sludge is $\mathrm{P}>50 \%$, the drying rate decreases with the increase of the thickness. When $\mathrm{P}<50.0 \%$, the drying rate increases with the increase of the thickness of the sludge cake. When the microwave is $160 \mathrm{~W}$, the drying effect of the sludge cake whose thickness is $1 \mathrm{~cm}$ is best.

By Figure 2, under the microwave condition of $320 \mathrm{~W}$, the shape of the dry rate curve of the sludge cake with the diameter of $3.0 \mathrm{~cm}$ and the thickness of $1.0 \mathrm{~cm}, 2.0 \mathrm{~cm}, 3.0 \mathrm{~cm}$ tends to be the same, which is constituted by three stages, that is acceleration, constant speed and deceleration. From the picture, when the moisture content of sludge is $\mathrm{P}>50 \%$, the drying rate decreases with the increase of the thickness. When $\mathrm{P}<50.0 \%$, the drying rate increases with the increase of the thickness of the sludge cake. 


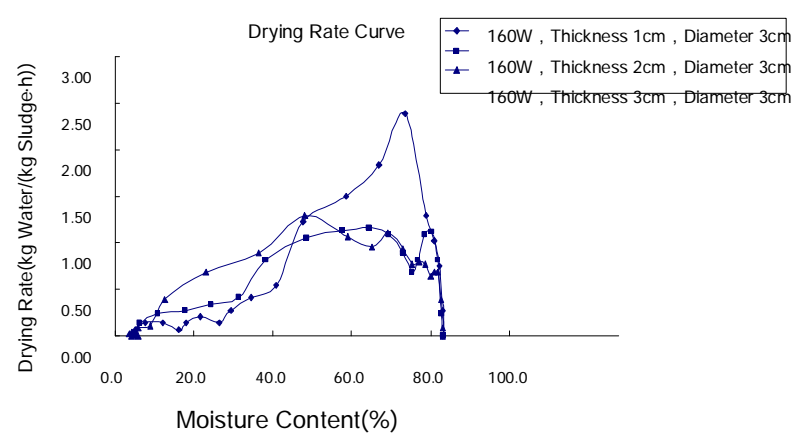

Fig.1 Rate curve to different thicknesses cake (160W)

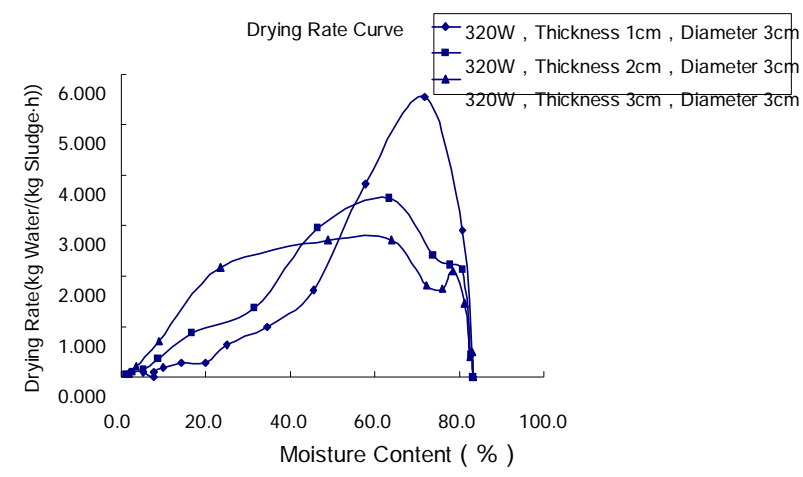

Fig.2 Rate curve to different thicknesses cake (320W)

By Figure 3, when the prerequisite of microwave is $480 \mathrm{~W}$, the shape of the dry rate curve of the sludge cake with the diameter of $3.0 \mathrm{~cm}$ and the thickness of $1.0 \mathrm{~cm}, 2.0 \mathrm{~cm}, 3.0 \mathrm{~cm}$ is consistent, which consist of three stages, that is acceleration $(70.0 \%<\mathrm{P}<83.3 \%)$, constant speed $(50.0 \%<\mathrm{P}<70.0 \%)$ and deceleration $(\mathrm{P}<50.0 \%)$. When the moisture content of sludge is $\mathrm{P}>50 \%$, the drying rate decreases with the increase of the thickness. When $\mathrm{P}<50.0 \%$, the drying rate increases with the increase of the thickness of the sludge cake. When the time of drying is up to 20min, the moisture content of the sludge cake whose thickness is $1.0 \mathrm{~cm}$ has dropped to $2.7 \%$, while the water content of the sludge's the thickness is $2.0 \mathrm{~cm}$ and $3.0 \mathrm{~cm}$ is each $3.9 \%$ and $6.8 \%$. When drying time of sludge cake is up to $22.5 \mathrm{~min}$, the moisture content of the sludge cake whose thickness is $2.0 \mathrm{~cm}$ has dropped to $1.3 \%$, and the moisture content of the sludge cake's thickness is $3.0 \mathrm{~cm}$ has dropped to $1.8 \%$, while the dry rate of the sludge cake's thickness is $3.0 \mathrm{~cm}$ is still very large, which is about twice the dry rate of the sludge cake's thickness is $2.0 \mathrm{~cm}$.

By Figure 4, when the prerequisite of microwave is $640 \mathrm{~W}$, the shape of dry rate curve of the sludge cake with the diameter of $3.0 \mathrm{~cm}$ and the thickness of $1.0 \mathrm{~cm}, 2.0 \mathrm{~cm}, 3.0 \mathrm{~cm}$ tends to be the same, which is comprise of three stages, that is acceleration $(70.0 \%<\mathrm{P}<83.3 \%)$, constant speed $(50.0 \%<\mathrm{P}<70.0 \%)$ and deceleration $(\mathrm{P}<50.0 \%)$. When the moisture content of sludge is $\mathrm{P}>50 \%$, the drying rate decreases with the increase of the thickness of sludge. When $\mathrm{P}<50.0 \%$, the drying rate increases with the increase of the thickness of the sludge cake. The effect of different power heating on the drying rate of sludge is different, but the effect of regularity is the same.

By Figure 5, when the qualification of microwave is $800 \mathrm{~W}$, the shape of dry rate of the sludge cake with the diameter of $3.0 \mathrm{~cm}$ and the thickness of $1.0 \mathrm{~cm}, 2.0 \mathrm{~cm}, 3.0 \mathrm{~cm}$ is consistent, which is comprise of three stages, that is acceleration $(70.0 \%<\mathrm{P}<83.3 \%)$, constant speed $(50.0 \%<\mathrm{P}<70.0 \%)$ and deceleration $(\mathrm{P}<50.0 \%)$. When the moisture content of sludge is $\mathrm{P}>50 \%$, the drying rate decreases with the increase of the thickness. When $\mathrm{P}<50.0 \%$, the drying rate increases with the increase of the thickness of the sludge cake.

Comprehensive analysis of experimental data can be drawn, in the $180 \mathrm{~W}, 320 \mathrm{~W}, 480 \mathrm{~W}, 640 \mathrm{~W}$, $800 \mathrm{~W}$ microwave conditions, when the moisture content of sludge is $\mathrm{P}>50 \%$, the drying rate decreases with the increase of the thickness; when $\mathrm{P}<50.0 \%$,the drying rate increases with the increase of the thickness of the sludge cake.

\section{The effect of sludge cake of different diameter on the drying rate of sludge}

First of all, make sludge into sludge cake with $3.0 \mathrm{~cm}$ in diameter and $1.0 \mathrm{~cm}, 2.0 \mathrm{~cm}$ and $3.0 \mathrm{~cm}$ in thickness, and each of these are two. Then puts these sludge into the six clean crucible which was burned to the constant weight. Thirdly, under the microwave condition of $480 \mathrm{~W}$, the mass of the sludge cake was quickly weighed at every two minutes. When the moisture content of wet basis reached about $2 \%$, the experiment was stopped. At last, calculate the rate of water content and drying of sludge cake in different diameter at each time point, and draw the sludge drying rate curve, 
as shown in figure 6.

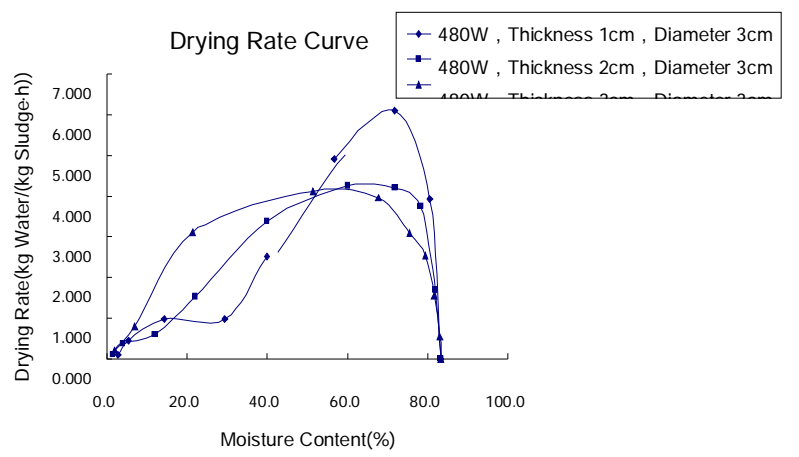

Fig.3 Rate curve to different thicknesses cake (480W)

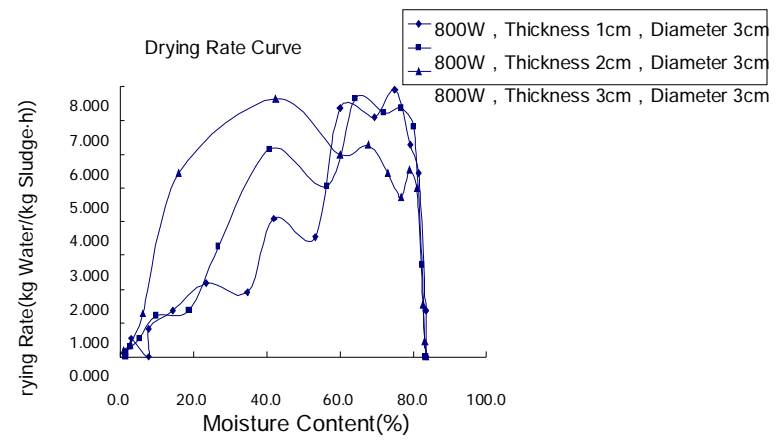

Fig.5 Rate curve to different thicknesses cake (800W)

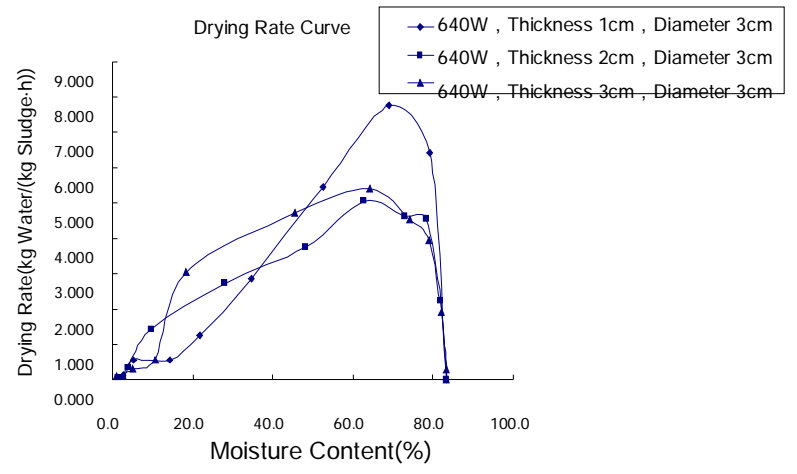

Fig.4 Rate curve to different thicknesses cake (640W)

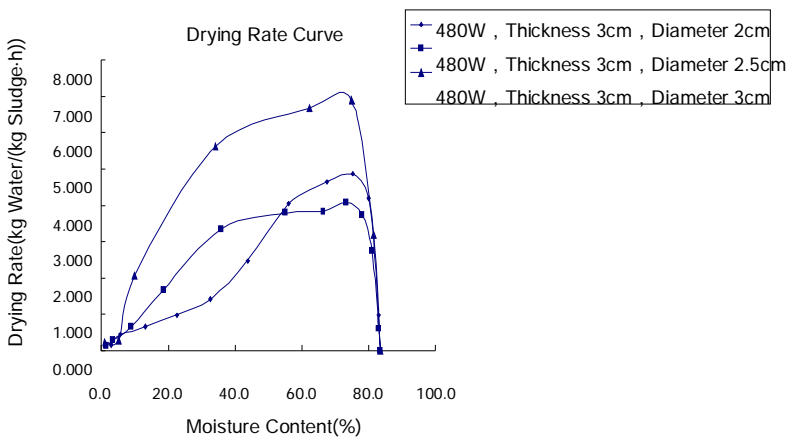

Fig.6 Drying rate curve to different diameter cake (480W)

By Figure 6, under the microwave condition of 480W, the dry rate of the sludge cake with the thickness of $3.0 \mathrm{~cm}$ and the diameter of $3.0 \mathrm{~cm}$ is higher than that of the sludge cake with the diameter of $2.0 \mathrm{~cm}$ and $2.5 \mathrm{~cm}$. In most cases, the dry rate of the sludge cake's diameter is $2.5 \mathrm{~cm}$ is higher than that of the diameter is $2.0 \mathrm{~cm}$. This shows that the drying rate increases with the increase of the diameter of the sludge.

Microwave heating is a method that the molecules obtain energy to achieve the effect of heating temperature by making use of high frequency electromagnetic wave to heat the material and then to change the transfer of the medium ion and the rotation of the dipole in order that the heat movement of the medium molecules is increased, and the friction between molecules is generated $^{[12]}$. The relative permittivity of general material is between 1 and 10 . While the relative dielectric constant of water is 78.54, which is much greater than that of dry matter. So water is the best medium to absorb microwave, which means most of the energy released by the electromagnetic field is absorbed by water molecules in the material and then converted to the latent heat of vaporization. So within the material, the process of heating and evaporation are carried out simultaneously. Due to the evaporation cooling, the surface temperature of the material is slightly lower than that of the internal temperature. Meanwhile, heat inside the generated material contributes to the internal steam generated rapidly, resulting in a pressure gradient. The higher the water content is, the effect of the pressure gradient on the exclusion of water is greater ${ }^{[13]}$. Thus, in the process of microwave drying, the transfer direction of the temperature gradient, heat transfer and steam pressure is consistent, which greatly improve the water transfer condition; Meanwhile, Swing to the existent of the power of pressure migration the microwave drying sludge gets the characteristics of drying from the inside to the outside---for overall material, inner material will be the first to be dried, which overcomes the shortcomings that for the first drying of outer material, shell plate forms to block the internal moisture to move outside ${ }^{[14-15]}$. That is "beggar's chicken 
effect". Therefore, when the same kind of wet sludge that is treated by different drying methods reach the same equilibrium moisture content, the microwave drying time is the shortest, and it has little relationship with the shape and size of the wet sludge. And the effect of microwave drying is better than that of conventional drying process. In addition, because inner dry sludge molecules are non polar, they don't directly absorb microwave energy in the process of drying, only to get the heat transfer of water or other wet sludge indirectly. So the temperature of the dry sludge is always lower than that of water or other wet sludge. Thus the dry material can get better drying effect when being dried at the setting temperature.

\section{Conclusions}

The process of microwave drying of the municipal sludge consists of three stages: rising, velocity and falling velocity; When the moisture content of the sludge is higher than $50 \%$ during the microwave drying, the drying rate decreases with the increase of the thickness of sludge. When is less than $50 \%$, the drying rate increases with the increase of the thickness of sludge. Microwave drying can greatly improve the condition of water translocation during the drying process. The greater the microwave power is, the greater the diameter is, and dry speed is faster.

\section{Acknowledgement}

This research was supported by a grant from National Natural Science Foundation of China(No.51208393), Hubei Department of Education Science Foundation (No.201304904) and the University Youth Grant(No.2012040). The authors would also like to thank the anonymous reviewers for their commenting of this paper.

\section{Reference :}

[1]Dao'an Zhou,Jin Huang,Hailong Bai,et al.. Experimental research on thermal drying and combustion characteristics of sludge[J].Environmental Pollution and Control,2012,4:5-10.

[2]Jinrong Qiu,Qitang Wu,Zebin Wei,et al.Research advances in municipal sludge drying[J].Ecology and Environment,2007,16(2):667-671.

[3]Rafd, Janb, Elisabethn.Fenton peroxidation improves the drying permance of waste activated sludge[J]. Journal of Hazardous Materials,2005,117:161-170.

[4]Jie Yu, Ningning Tian,et al. Analysis and discussion of sludge disposal and treatment of sewage treatment plants in China[J]. Chinese Journal of Environmental Engineering, 2007, 1(1):82-86.

[5]Jianming Ke, Kaijun Wang, Ningning Wang. Study on treatment and disposal of municipal sewage sludge in Beijing[J].China Biogas, 2000,18(3): 35-38.

[6]Fytili D, Zabaniotou A.Utilization of sewage sludge in EU application of old and new methods-A review[J].Renewable and Sustainable Energy Reviews,2008, 12:116-140.

[7]MGrath S P , C hang A C , Page A L, et al. Land application of sewage sludge :scientific perspectives of heavy metal loading limits in Europe and the United States[J]. Environment. Resource, 1994 , 2:108-118.

[8]Cyr M, Coutand M, Clastres P. Technological and environmental behavior of sewage sludge ash (SSA) in cement-based materials[J]. Cement and Concrete Research, 2007, 37:1278-1289.

[9]Long Wu, Zhonghua Wu, Zhanyong Li,et al.Analysis of Energy Consumption in Isothermal Hot Air Convective Drying Process of Sewage Sludge[J].Chian Water \& Wasterwater,2013,29(15) : 259-267. 
[10]Kim Y, Parker W.A technical and economic evaluation of the pyrolysis of sewage sludge for the Production of bio-oil[J]. Bioresource Technology, 2008, 99:1409 1416.

[11]Hall J E. Sewage sludge production treatment and disposal in the European Union[J]. J CIWEM,1995,19(8): 335-343.

[12]Yanli Qu,Ai'min Li,Rundong Li,et al. Study on Drying Characteristic of Caky Sludge[J]. Journal of Combustion Science and Technology,2005,11(1) : 97-99.

[13]Stelmach S, Wasielewski R. Co-combustion of dried sewage sludge and coal in a pulverized coal boiler[J].Journal of Material Cycles Waste Management[J], 2008, 10:110-115.

[14]Vaxelaire J.Puiggali J R.Analysis of the Drying of Residual Sludge:From the Experiment to the Simulation of a Belt Dryer[J]. Drying Technology,2002,20(4 5): 989-1008.

[15]Luyi Zou, Jianhong Ju. Preliminary study on microwave dehydration of activated sludge[J]. Journal of Jiangnan College,1998,13(4):56-60. 